\title{
Surgical treatment of dumbbell-shaped jugular foramen schwannomas
}

\author{
Paulo A. S. Kadri, M.D., And Ossama Al-Mefty, M.D. \\ Department of Neurosurgery, University of Arkansas for Medical Sciences, Little Rock, Arkansas
}

\begin{abstract}
Object. Schwannomas of the jugular foramen are rare, comprising between 2 and $4 \%$ of intracranial schwannomas. The authors retrospectively analyzed their surgical experience with schwannomas of the lower cranial nerves that presented with intra- and extracranial extensions through an enlarged jugular foramen. The transcondylar suprajugular approach was used without sacrificing the labyrinth or the integrity of the jugular bulb. In this report the clinical and radiological features are discussed and complications are analyzed.

Methods. This retrospective study includes six patients (three women and three men, mean age 31.6 years) with dumbbell-shaped jugular foramen schwannomas that were surgically treated by the senior author during a 5.5-year period. One patient had undergone previous surgery elsewhere. Glossopharyngeal and vagal nerve deficits were the most common signs (appearing in all patients), followed by hypoglossal and accessory nerve deficits (66.6\%). Two or more signs or symptoms were present in every patient. Three tumors presented with cystic degeneration. In four patients the jugular bulb was not patent on neuroimaging studies. The suprajugular approach was used in five patients; the origin of the tumor from the 10th cranial nerve could be defined in three of them. All lesions were completely resected. No death or additional postoperative cranial nerve deficits occurred in this series. Aspiration pneumonia developed in one patient. Preoperative deficits of the ninth and 10th cranial nerves improved in one third of the patients and half recovered mobility of the tongue. No recurrence was discovered during the mean follow-up period of 32.8 months.

Conclusions. With careful, extensive preoperative evaluation and appropriate planning of the surgical approach, dumbbell-shaped jugular foramen schwannomas can be radically and safely resected without creating additional neurological deficits. Furthermore, recovery of function in the affected cranial nerves can be expected.
\end{abstract}

\section{KEY WORDS • schwannoma • brain neoplasm • jugular foramen • lower cranial nerve $\bullet$ skull base $・$ surgical approach}

Although they can be located along any cranial, spinal, or peripheral nerve, $25 \%$ of schwannomas are found in the head and neck..$^{19}$ Intracranial schwannomas that arise from sites other than the vestibular nerves are rare, constituting 2.9 to $4 \%$ of such lesions. ${ }^{16,35}$ Of these, the highest number occur in the trigeminal nerve (40\%), followed by the facial nerve (23\%), and the lower cranial nerves $(20 \%)$. $^{16,32}$ Schwannomas located at the jugular foramen may arise from the glossopharyngeal, vagus, or accessory nerve. The proximity and clinical manifestation of schwannomas originating from the hypoglossal nerve are the reason some authors classify these tumors with jugular foramen schwannomas. These tumors can also originate from the cisternal, foraminal, or extracranial portion of the lower cranial nerves. Tumors originating from the cisternal portion present with major intracranial growth; tumors originating from the foraminal portion predomi-

Abbreviations used in this paper: $\mathrm{CA}=$ carotid artery; $\mathrm{CT}=$ computerized tomography; MR = magnetic resonance. nantly expand the bone, and tumors originating from the distal portion present with major extracranial growth. ${ }^{20,22}$

One subset of jugular foramen schwannomas presents with both extra- and intracranial growth through an enlarged jugular foramen. These are called dumbbell- or saddlebag-shaped tumors. ${ }^{29,32}$ Recent advancements in microneuroanatomy, neuroradiology, and microsurgical and skull base techniques have substantially improved the outcome in patients with these tumors, and authors of recent studies have reported no deaths directly related to the surgical procedure. ${ }^{10,12-15,17,20,22,32,33,35}$ Nonetheless, the real challenge for neurosurgical treatment is to preserve the function of the lower cranial nerves while achieving radical resection and decreasing the risk of recurrence. These challenges are found especially in patients with dumbbellshaped tumors in which the cranial nerves are at risk during resection of the intracranial, intraforaminal, and extracranial sections. We present the experience of the senior author (O.A.) with the treatment of dumbbell-shaped jugular foramen schwannomas, stressing the importance of 
perioperative precautions and the details of the surgical methods used.

\section{CLINICAL MATERIAL AND METHODS}

We retrospectively analyzed a series of six patients with schwannomas in whom we found intra- and extracranial extensions of tumor through the jugular foramen. These six patients had been treated by the senior author in the past 5.5 years (December 1998-April 2004). As shown in Table 1, we excluded those with schwannomas of the lower cranial nerves that had no intra- and extracranial extensions (Type D according to the classification system of Pellet, et al., ${ }^{29}$ modified from Kaye, et al. ${ }^{20}$ ). We also excluded patients with schwannomas of the hypoglossal nerve and those with neurofibromatosis Type 2. The medical charts, follow-up data, and neuroimages obtained in each patient were reviewed.

\section{Demographic Data}

The most important demographic, clinical, and histological features in our series of patients are outlined in Table 2. Patients in the study group ranged in age from 23 to 42 years (mean 31.6 years); the series consisted of three women and three men. The lesion was located on the right side in four patients $(66.6 \%)$ and on the left side in two (33.3\%). Symptoms developed in one patient at 16 weeks of pregnancy and she underwent surgery after delivery of her baby. One patient presented with marked hydrocephalus, and a ventriculoperitoneal shunt was placed before surgical exploration was performed. One patient had undergone a partial resection at another institution, during which the sinus was ligated. The duration of the follow-up period ranged from 2 to 67 months (mean 32.8 months).

\section{Presenting Symptoms, Signs, and Findings}

The most common presenting symptoms were dysphagia and dysphonia (four patients), followed by weakness of the shoulder and gait disturbance (two patients). Hearing loss, tinnitus, dizziness, headache, seizures, and diplopia were present in one patient each. Preoperative glossopharyngeal and vagal deficits were present in all patients. Hypoglossal and accessory nerve deficits were present in four patients (66.6\%), and loss of hearing was diagnosed in two (33.3\%). Hypalgesia in the territory of the second division of the trigeminal nerve, lateral gaze paralysis, facial paralysis (Grade 3/6 according to the House-Brackmann classification) and cerebellar dysfunction were present in one patient each. Two or more signs or symptoms were present in every patient. One patient had hydrocephalus and underwent shunt implantation before tumor removal.

\section{Preoperative Protocol}

We used the same preoperative protocol as for other lesions in the jugular foramen. ${ }^{6}$ The following is summary of that protocol.

Neuroimaging. Preoperative MR imaging with and without contrast enhancement, angiography or arteriovenous MR angiography, and very thin slices of CT scans constituted the radiological work-up used to explore the

TABLE 1

Demographic data in six patients with jugular foramen schwannomas*

\begin{tabular}{|c|c|c|c|c|c|c|c|}
\hline $\begin{array}{l}\text { Case } \\
\text { No. }\end{array}$ & $\begin{array}{l}\text { Age (yrs), } \\
\text { Sex }\end{array}$ & Side & Preop Symptoms & Preop Signs & $\begin{array}{l}\text { Nerve of } \\
\text { Origin }\end{array}$ & Postop Status & $\mathrm{FU}(\mathrm{mos})$ \\
\hline $2 \dagger$ & $30, \mathrm{~F}$ & $\mathrm{rt}$ & $\begin{array}{l}\text { decreased hearing, } \\
\text { tinnitus, gait dist }\end{array}$ & $\begin{array}{l}\text { rt 8th, 10th, \& } \\
\text { 12th CNs }\end{array}$ & 10th CN & no new deficit & $\begin{array}{l}\text { 66; hearing imp, } \\
\text { 10th \& 12th CNs }\end{array}$ \\
\hline 3 & $42, \mathrm{~F}$ & $\mathrm{rt}$ & $\begin{array}{l}\text { dysphagia, dysphonia, } \\
\text { gait dist }\end{array}$ & $\begin{array}{l}\text { rt } 7 \text { th }(3 / 6 \mathrm{HB}), \\
8 \text { th (mild), 9th, } \\
\& 10 \text { th CNs }\end{array}$ & 10th CN & no new deficit & $\begin{array}{l}\text { 42; medial of vocal } \\
\text { cord, complete } \\
\text { recovery from } \\
\text { facial paralysis }\end{array}$ \\
\hline $4 \ddagger$ & $23, \mathrm{~F}$ & lt & $\begin{array}{l}\text { seizures, vertigo, } \\
\text { dysphonia }\end{array}$ & $\begin{array}{l}\text { gait ataxia, } \\
\text { 1t V2 div } \\
\text { (hypalgesia) \& } \\
\text { 9th, 10th, 11th, } \\
\& 12 \text { th CNs }\end{array}$ & 10th CN & no new deficit & 36 ; stable \\
\hline 5 & $33, \mathrm{M}$ & lt & $\begin{array}{l}\text { headaches, dysphagia, } \\
\text { dysphonia, shoulder } \\
\text { weakness }\end{array}$ & $\begin{array}{l}\text { It } 9 \text { th, } 10 \text { th, } \\
\text { \& 11th CNs }\end{array}$ & ND & no new deficit & 24; stable \\
\hline $6 \S$ & $30, \mathrm{M}$ & lt & $\begin{array}{l}\text { double vision, } \\
\text { dysphagia }\end{array}$ & $\begin{array}{l}\text { 1t 6th, 9th, 10th, } \\
11 \text { th, \& 12th CNs }\end{array}$ & ND & no new deficit & $\begin{array}{l}\text { 23; imp, 6th, 9th, } \\
\text { \& 12th CNs, } \\
\text { swallowing back } \\
\text { to normal (10th } \\
\text { CN), persistent } \\
\text { dysphonia }\end{array}$ \\
\hline 7 & $32, \mathrm{M}$ & lt & $\begin{array}{l}\text { dysphagia, dysphonia, } \\
\text { atrophy of lt shoulder }\end{array}$ & $\begin{array}{l}\text { It } 9 \text { th, } 10 \text { th, } 11 \text { th, } \\
\text { \& } 12 \text { th CNs }\end{array}$ & ND & no new deficit & $2 ;$ stable \\
\hline
\end{tabular}

$* \mathrm{CN}=$ cranial nerve; dist = disturbance; $\mathrm{FU}=$ follow up; $\mathrm{HB}=$ House - Brackmann; imp = improvement; medial = medialization; ND $=$ not defined.

$\dagger$ Patient presented with symptoms during pregnancy; surgery performed after cesarean section.

$\ddagger$ Previous surgery performed at another hospital.

$\S$ Hydrocephalus requiring a preoperative ventriculoperitoneal shunt. 
TABLE 2

Classification system for jugular foramen schwannomas*

\begin{tabular}{ll}
\hline \hline Class & Definition \\
\hline Type A & primarily intracranial: minimal extension into jugular foramen \\
Type B & primarily intraosseous: w/ or w/o intracranial extension \\
Type C & primarily extracranial: minimal extension into jugular foramen \\
Type D & saddlebag- or dumbbell-shaped intra- \& extracranial extensions \\
\hline
\end{tabular}

* Proposed by Pellet, et al.

anatomy of the jugular foramen and fossa, the temporal bone, and the condyles. The nature of the tumor (cystic or solid), extensions (intracranial, extracranial, or dumbbell), and the characteristics of bone involvement (the presence of sclerosis and enlargement of the canal) were studied using MR images and CT scans with the aid of a bone algorithm. The dominant vertebral artery and the characteristics of the vertebrobasilar system were studied. Special attention was paid to the venous phase in terms of the size, dominance, and tributaries (superior petrosal, inferior petrosal, and vein of Labbé) of the transverse and sigmoid sinuses, and the position and size of the jugular bulb. Intraoperative image-guided frameless stereotaxy was an important tool used during the surgical procedure. Postoperative imaging included a CT scan obtained 24 hours after surgery and MR images obtained during the early postoperative period, followed by MR images acquired at 3 months, 6 months, and then annually.

Assessment of Lower Cranial Nerves. Extensive audiological and otolaryngological evaluations were done. Speech evaluations, audiological discrimination, and swallowing studies were conducted before and after surgery. Feeding by mouth was allowed only if the postoperative swallowing studies showed satisfactory results, assuring that the function of the lower cranial nerves could protect the airway. Otherwise, the patient was kept on a nothing-by-mouth regimen and appropriate precautions were taken.

Intraoperative neurophysiological monitoring of the 10th cranial nerve was accomplished using an electromyographic endotracheal tube. Electromyographic needles were inserted into the mimic muscles, the sternocleidomastoid muscle, and the tongue to study the seventh, 11 th, and 12th cranial nerves, respectively.

\section{Surgical Approach}

For lesions located within the jugular foramen, we use variations of the transcondylar approach, ${ }^{6}$ corresponding to the suprajugular, the retrojugular, and the transjugular approaches. ${ }^{1-3,5-8}$ Schwannomas differ from glomus tumors and meningiomas located within the jugular foramen and fossa because they compress rather than invade the jugular bulb, and the nerves of origin are positioned anterior to this structure. Therefore, we used the transcondylar suprajugular approach without sacrificing the labyrinth or the integrity of the wall of the jugular bulb. Even if preoperative studies revealed an absence of flow into the jugular bulb, the transjugular approach was not used because the sinus usually recovers its patency after the decompression.

\section{Suprajugular Approach}

The suprajugular approach is essentially a presigmoid infralabyrinthine route. We use this approach if the tumor extends anteriorly to the jugular bulb. A postauricular incision is made, and the internal CA, external CA, internal jugular vein, and the ninth, 10th, 11th, and 12th cranial nerves in the cervical region are identified. The sternocleidomastoid muscle is dissected, mobilized, and reflected inferiorly. A mastoidectomy is followed by complete skeletonization of the sigmoid sinus, jugular bulb, and jugular vein. The presigmoid, infralabyrinthine space is exposed and the dura mater is identified superior to the patent jugular bulb and inferior to the labyrinth. After the cerebellomedullary cistern is opened, releasing cerebrospinal fluid, the tumor is exposed. The lesion is debulked with suction and bipolar coagulation or by using the ultrasonic aspirator. The lower cranial nerves (ninth-12th), the posteroinferior cerebellar artery, the anteroinferior cerebellar artery, and the vertebral artery are dissected away from the tumor through the arachnoid plane, and the lesion is radically removed.

\section{RESULTS}

\section{Imaging Characteristics}

Admission MR images were available for all patients. The venous and arterial anatomy was studied using angiography (three patients) and/or venous and arterial phase MR angiography (four patients). Preoperative CT scans obtained with detailed bone algorithms were available for five patients. The tumors were classified as Type D according to the Pellet classification (Table 2); each presented with an intra- and extracranial extension.

All lesions had a low to isointense signal on $\mathrm{T}_{1}$-weighted images and a high signal on $\mathrm{T}_{2}$-weighted images. Five tumors showed marked enhancement and one had moderate enhancement after Gd contrast injection. Three tumors revealed cystic degeneration on MR imaging studies. All lesions were hypo- or avascular on conventional (three patients) or MR angiography (four patients). Preoperative embolization was not attempted in any of the patients. In four cases, the jugular bulb was not patent on the angiographic studies. In all patients an enlarged jugular foramen with well-delineated sclerotic margins was observed on thin-cut CT scans with bone algorithms (Fig. 1).

\section{Postoperative Course}

The tumors were completely resected in all patients, and none experienced additional cranial nerve deficits. One patient had swelling of the neck on the 1st postoperative day and a tracheotomy was performed to secure the airway. The results of radiological examinations were consistent with fluid accumulation, most likely cerebrospinal fluid. This patient had presented with preoperative ninth and 10th cranial nerve deficits. Despite all efforts to avoid aspiration, the patient suffered aspiration pneumonia but was successfully treated, and the fluid accumulation resolved spontaneously. Although this patient reported no dysphagia during follow up and swallowing studies showed no signs of aspiration, a medialization of the vocal cord with Teflon was done to improve the dysphonic symptoms. 

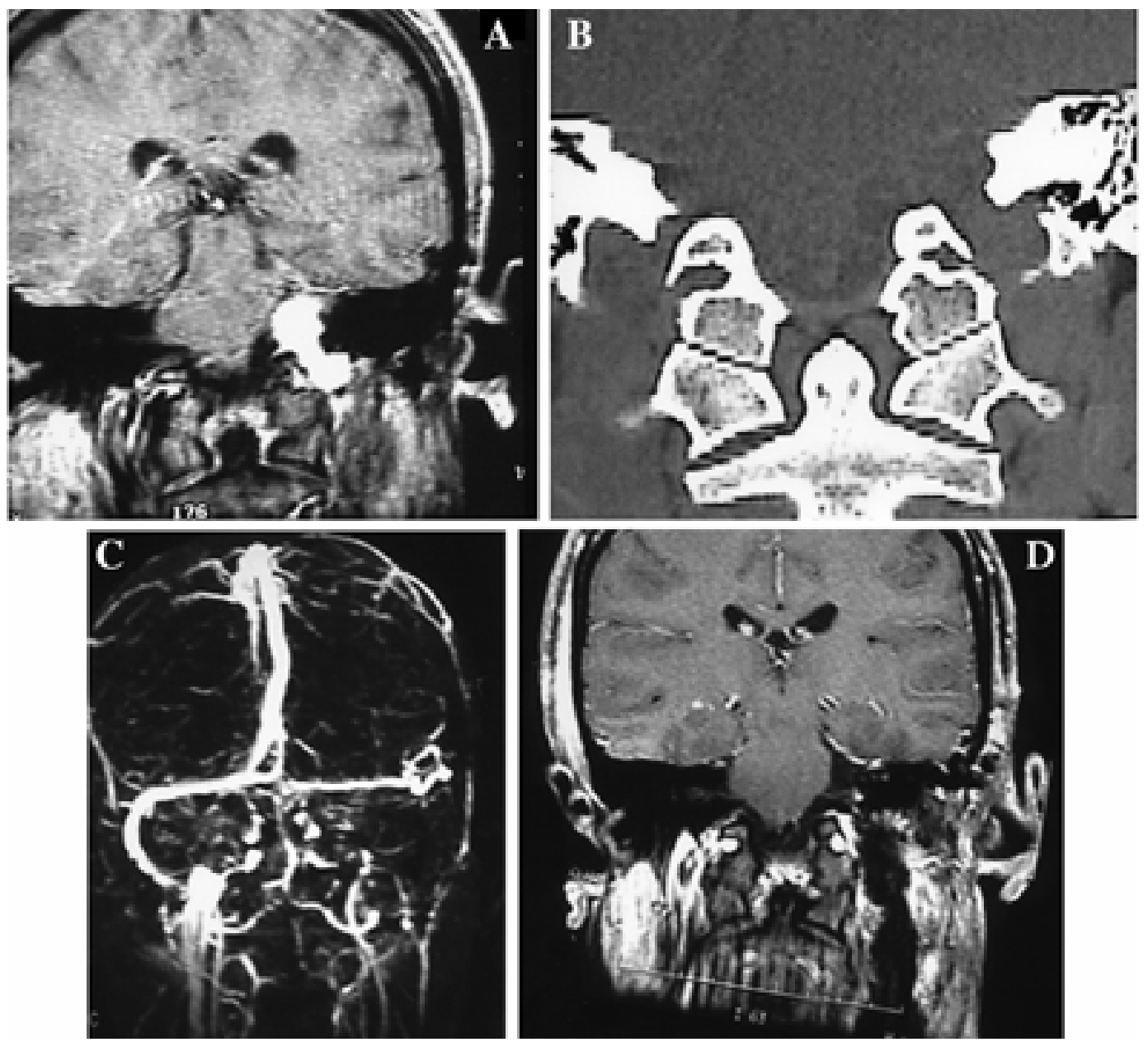

Fig. 1. A and B: Preoperative coronal enhanced MR image (A) and bone CT scan (B) demonstrating a dumbbellshaped jugular foramen schwannoma. C: Admission MR venous angiography revealing occlusion of the left sigmoid sinus. D: Postoperative MR image (fat suppression) obtained after complete resection of the tumor.

Two $(33.3 \%)$ of the six patients with preoperative ninth and 10th cranial nerve palsy attained significant improvement of their deficits. Two (50\%) of four patients with preoperative hypoglossal nerve palsy completely recovered mobility of the tongue. One of the two patients with hearing loss had significant improvement of that deficit, and the patient with facial palsy completely recovered nerve function. The patient with a sixth cranial nerve deficit completely recovered ocular motion, and the condition of the remaining patients was unchanged postoperatively. To date, none of the patients have shown signs of recurrence (follow-up period 2-66 months, mean 32.8 months).

\section{DISCUSSION}

\section{Clinical Presentation and Classification}

A variety of lesions can arise from the structures normally found within the jugular foramen and fossa or from contiguous structures. Although they are rare, the three most common diagnoses of mass lesion within the jugular foramen are glomus jugulare tumors, schwannomas, and meningiomas. Differences in the treatment plans for each of these lesions make a preoperative diagnosis essential.
Glomus jugulare tumors are managed with preoperative embolization, and meningiomas must be treated by maximal removal of bone and involved dura mater.

The most common lesion is the glomus jugulare tumor, which comprises 60 to $80 \%$ of primary tumors of the jugular foramen. ${ }^{25}$ Schwannomas of the jugular foramen are rare: Eldevik, et al., ${ }^{11}$ noted that approximately 130 cases not associated with neurofibromatosis have been reported. Arnautovic and Al-Mefty ${ }^{6}$ found fewer than 50 cases of jugular fossa meningioma reported in the literature. Other important differential diagnoses to be considered are acoustic schwannomas $4,27,28,33,34$ and other lesions such as chordomas, choroid plexus papillomas, chondromas, carcinomas, metastases, hemangioblastomas, epidermoid tumors, lymphomas, salivary gland tumors, pseudomasses such as normal vascular asymmetry, a high jugular bulb or jugular diverticulum, and aneurysms of the petrous CA. $., 18,26,32,35$

The clinical presentation of jugular foramen schwannomas depends on the tumor's extension. It is believed that the diagnosis and nerve origin can be determined by the initial symptoms and the grade of dysfunction of the nerve, especially when compared with adjacent nerves. 
This connection gives rise to the classic descriptions of the different syndromes affecting the lower cranial nerves, such as the ones discussed in Hakuba, et al., ${ }^{15}$ including descriptions by Vernet (involvement of the ninth, 10th, and 11th cranial nerves), Avellis (the 10th nerve), ColletSicard (the 11th and 12th nerves), Jackson (the 10th, 11th, and 12th nerves), and Tapia (the 10th and 12th nerves) as well as a combination of these syndromes.

Rather than corresponding to the nerve of origin, however, the symptoms are marked by their variability in presentation, and some patients have no dysfunction of the lower cranial nerves. Symptoms secondary to increased cranial pressure (blurred vision and headaches) are actually the most common complaints, and the most common and earliest sign is compromise of the eighth cranial nerve complex. This is what makes the differential diagnosis of schwannomas and acoustic tumors difficult. ${ }^{4}$, 9, 19,22,24,27,28,32,33,35 The presence of nystagmus, ataxia, vertigo, pyramidal tract involvement, and other cranial nerve deficits (fifth, sixth, and seventh nerves) has been reported. ${ }^{10}$, ${ }_{12-15,17,19,20,21,23,33,35}$ Hakuba, et al., ${ }^{15}$ divided the clinical symptoms into four categories: cranial nerve involvement, unilateral cerebellar involvement, nystagmus, and signs or symptoms secondary to intracranial pressure.

Usually the tumor does not manifest clinically until it reaches a fairly large size. Kinney, et al., ${ }^{22}$ correlated the position of the tumor with the nerve's point of origin: tumors originating proximally present with intracranial growth, those originating in the midregion expand into the bone, and lesions originating distally present with extracranial growth. Based on this characteristic, Kaye, et al. ${ }^{20}$ proposed a classification in which primarily intracranial tumors with only a small extension into the bone are called Type A; tumors in which the main mass is within the bone, with or without an intracranial component, are called Type B; and tumors that are primarily extracranial with only a minor extension into the bone are called Type C. Pellet, et al., ${ }^{29}$ described one case of jugular foramen schwannoma in which there was both an extra- and an intracranial extension, thereby adding a Type D for saddlebag-shaped or dumbbell tumors.

Type A tumors cause symptoms of a posterior fossa mass, mimicking acoustic neuromas, with minimal if any manifestation of lower cranial nerve deficits, whereas tumors expanding into the bone or upper cervical region cause earlier compression of the nerves within the jugular foramen. ${ }^{20}$ In a review of the literature published since the MR imaging era began, Kanemoto, et al., ${ }^{18}$ found that $67 \%$ of tumors primarily located intracranially caused hearing loss, whereas significantly fewer patients with extracranial or intraforaminal tumors had eighth cranial nerve deficits. Only one of our patients lost hearing, and none had a main intracranial component of the tumor. It is not always possible to identify the origin of these lesions. ${ }^{18}$ It is believed, however, that the glossopharyngeal nerve is the most common origin for tumors with a mainly intracranial extension, whereas the vagus nerve is the origin for tumors with an extracranial extension..$^{20}$ In our series, the vagus nerve was identified as the nerve of origin in three of the six patients with Type D tumors.

\section{Neuroimaging-Confirmed Diagnosis}

In the past, neuroimaging examination has included plain x-ray films, CT scanning, pneumoencephalography, nuclear resonance scanning, and angiography. ${ }^{4,10,12,13 \text {, }}$ 15,19,23,27,28 As pointed out by Tan, et al. ${ }^{35}$ early diagnosis increases surgical safety and the chance to preserve the function of the lower cranial nerves. Preoperative MR imaging and CT scanning are important for diagnosis and to classify the tumor and plan the best surgical approach.

Thin CT scans $(1-3 \mathrm{~mm})$ performed with a detailed bone algorithm and coronal reconstruction provide adequate information about the position of the jugular bulb and enlargement of the canal, which usually appears as a sharp, rounded shape with sclerotic rims (bone scalloping). ${ }^{9,11,15,20}$ Erosion of the posterior margin of the internal meatus can also be observed. ${ }^{11}$ Such findings help to differentiate schwannomas from meningiomas and glomus jugulare tumors. Meningiomas frequently invade the bone, producing hyperostosis and thickness of the jugular spine and jugular tubercle. ${ }^{6}$ Glomus jugulare tumors tend to erode and destroy the bone, particularly the jugular spine and the carotid crest (caroticojugular spine).

Diagnostic MR imaging, with its multiplanar capabilities and the use of fat suppression techniques, is essential in determining the extension of the tumor intra- and extracranially. The use of MR imaging also reveals the tumor's characteristics, such as the presence of cystic components. ${ }^{24}$ These tumors have the same characteristics as other intracranial schwannomas on MR images, with a low $\mathrm{T}_{1}$ signal, a high $\mathrm{T}_{2}$ signal, and moderate to marked contrast enhancement. ${ }^{11,24,34}$ There is a lack of flow void, which is typical of glomus jugulare tumors and indicates their rich vascular network. There is absence of calcifications and dural tail, which are typical of meningiomas. Whereas glomus jugulare tumors and meningiomas tend to invade the lumen of the sinus and vein, sometimes showing intraluminal growth, schwannomas tend to compress the bulb and the jugular vein. ${ }^{24}$

\section{Treatment Methods}

Successful treatment of lesions involving the jugular foramen includes complete tumor removal without creating additional neurological deficits. Although in early surgical series ${ }^{30}$ a mortality rate as high as $16 \%$ had been reported, recent advances in surgical microneuroanatomy, neuroradiology, and microsurgical and skull base techniques have drastically decreased this rate, and no deaths directly related to the surgical procedure have been reported in the latest series. . $^{10,12-15,17,20,22,32,33,35}$ The lower cranial nerves must be carefully evaluated, because they are the source of major and life-threatening postoperative complications, especially if the patient had no deficits preoperatively. The acute development of postoperative deficits in such patients allows no chance for compensatory mechanisms to develop. ${ }^{32}$ Speech pathology and otolaryngological evaluations with pre- and postoperative swallowing, as well as audiological studies, are essential in the treatment of these patients. Appropriate measures, such as the nothing-by-mouth regimen with parenteral nutrition, swallowing exercises, and soft mechanical diets with swallowing precautions, are taken if the patient exhibits a risk of aspiration on postoperative swallowing studies. Postoperative deficits might improve, but if the risk of aspiration persists or the patient has marked dysphonia, vocal cord medialization is advised. 
The surgical approach should be well planned to achieve complete resection, because repeated operation drastically increases the chance of injury to the lower cranial nerves. Intraoperative electrophysiological monitoring of these nerves as well as brainstem auditory evoked potentials are important adjuvants for preventing injury to the lower cranial nerves, the facial nerve, and the vestibulocochlear complex. Frameless stereotactic navigation is also helpful, especially in the intraosseous portion of the tumor.

The choice of surgical approach is of vital importance for success. A safe exposure demands that the surgeon understand the microanatomy of the region to preserve the neurovascular structures. The tumor's location and extension defines the surgical approach. For tumors confined intracranially (Type A), a retrosigmoid approach provides adequate exposure. ${ }^{32}$ For tumors involving the bone window, a wider exposure is desirable. We do not favor sacrifice of the labyrinth to gain exposure, ${ }^{29}$ because improvements in hearing have been reported after removal of these tumors. ${ }^{10,28,32,36,37}$ Because schwannomas within the jugular foramen tend to displace the jugular bulb posteriorly, the suprajugular approach allows the surgeon to remove the tumor without opening the wall of the bulb.

The experience with radiosurgical treatment of jugular foramen schwannomas is still limited. ${ }^{21,25,31}$ In the largest reported series, Muthukumar, et al. ${ }^{26}$ concluded that this treatment should be reserved for patients with small tumors and intact lower cranial nerves. We prefer to reserve radiosurgical treatment for the rare patients in whom the venous anatomy presents a considerable risk, such as when there is a single functioning ipsilateral sigmoid sinus and jugular bulb.

\section{CONCLUSIONS}

Schwannomas of the lower cranial nerves are a rare subgroup of intracranial schwannomas. Dumbbell-shaped tumors tend to cause lower cranial nerve deficits rather than symptoms of increased intracranial pressure or hearing loss. With appropriate preoperative evaluation and careful planning of the perioperative period, these tumors can be safely removed and the neurovascular structures preserved without creating new neurological deficits. Functional recovery of preoperative deficits can be expected after total tumor removal.

\section{References}

1. Al-Mefty O: Operative Atlas of Meningiomas. Philadelphia: Lippincott-Raven, 1998, pp 209-348

2. Al-Mefty O, Fox JL, Rifai A: A combined infratemporal and posterior fossa approach for the removal of giant glomus tumors and chondrosarcomas. Surg Neurol 28:423-431, 1987

3. Anand VK, Leonetti JP, Al-Mefty O: Neurovascular considerations in surgery of glomus tumors with intracranial extensions. Laryngoscope 103:722-728, 1993

4. Arenberg IK, McCreary HS: Neurilemmoma of the jugular foramen. Laryngoscope 81:544-557, 1971

5. Arnautovic KI, Al-Mefty O: Foramen magnum meningiomas, in Kaye AH, Black PM (eds): Operative Neurosurgery. London: Churchill Livingstone, 2000, pp 623-634

6. Arnautovic KI, Al-Mefty O: Primary meningiomas of the jugular fossa. J Neurosurg 97:12-20, 2002
7. Arnautovic KI, Al-Mefty O, Husain M: Ventral foramen magnum meningiomas. J Neurosurg (Spine 1) 92:71-80, 2000

8. Borba LAB, Al-Mefty O: Paragangliomas of the skull base. Neurosurg Q 5:256-277, 1995

9. Caldemeyer KS, Mathews VP, Azzarelli B, et al: The jugular foramen: a review of anatomy, masses, and imaging characteristics. Radiographics 17:1123-1139, 1997

10. Clemis JD, Noffsinger D, Derlacki EL: A jugular foramen schwannoma simulating an acoustic tumor with recovery of retrolabyrinthine cochleovestibular function. Trans Am Acad Ophthalmol Otolaryngol 84:ORL-687-696, 1977

11. Eldevik OP, Gabrielsen TO, Jacobsen EA: Imaging findings in schwannomas in the jugular foramen. AJNR 21:1139-1144, 2000

12. Fink LH, Early CB, Bryan RN: Glossopharyngeal schwannomas. Surg Neurol 9:239-244, 1978

13. Franklin DJ, Moore GF, Fisch U: Jugular foramen peripheral nerve sheath tumors. Laryngoscope 99:1081-1087, 1989

14. Graham MD, LaRouere MJ, Kartush JM: Jugular foramen schwannomas: diagnosis and suggestions for surgical management. Skull Base Surg 1:34-38, 1991

15. Hakuba A, Hashi K, Fujitani K, et al: Jugular foramen neurinomas. Surg Neurol 11:83-94, 1979

16. Ho KL: Schwannoma of the trochlear nerve: case report. J Neurosurg 55:132-135, 1981

17. Horn KL, House WF, Hitselberger WE: Schwannomas of the jugular foramen. Laryngoscope 95:761-765, 1985

18. Kanemoto Y, Ochiai C, Yoshimoto Y, et al: Primary extracranial jugular foramen neurinoma manifesting with marked hemiatrophy of the tongue: case report. Surg Neurol 49:534-537, 1998

19. Katz AD, Passy V, Kaplan L: Neurogenous neoplasms of major nerves of face and neck. Arch Surg 103:51-56, 1971

20. Kaye AH, Hahn JF, Kinney SE, et al: Jugular foramen schwannomas. J Neurosurg 60:1045-1053, 1984

21. Kida Y, Kobayashi T, Tanaka T, et al: [A new strategy for the treatment of jugular foramen tumors using radiosurgery.] No Shinkei Geka 23:671-675, 1995 (Jpn)

22. Kinney SE, Dohn DF, Hahn JF, et al: Neuromas of the jugular foramen, in Brackmann DE (ed): Neurological Surgery of the Ear and Skull Base. New York: Raven Press, 1982, pp 361-368

23. Maniglia AJ, Chandler JR, Goodwin WJ Jr, et al: Schwannomas of the parapharyngeal space and jugular foramen. Laryngoscope 89:1405-1414, 1979

24. Matsushima T, Hasuo K, Yasumori K, et al: Magnetic resonance imaging of the jugular foramen neurinomas. Acta Neurochir 96:83-87, 1989

25. Megerian CA, McKenna MJ, Nadol JB Jr: Non-paraganglioma jugular foramen lesions masquerading as glomus jugulare tumors. Am J Otol 16:94-98, 1995

26. Muthukumar N, Kondziolka D, Lunsford LD, et al: Stereotactic radiosurgery for jugular foramen schwannomas. Surg Neurol 52:172-179, 1999

27. Naunton RF, Proctor L, Elpern BS: The audiologic signs of ninth nerve neurinoma. Arch Otolaryngol 87:222-227, 1968

28. Neeley JG: Reversible compression neuropathy of the eighth cranial nerve from a large jugular foramen schwannoma. Arch Otolaryngol 105:555-560, 1979

29. Pellet W, Cannoni M, Pech A: The widened transcochlear approach to jugular foramen tumors. J Neurosurg 69:887-894, 1988

30. Pluchino F, Crivelli G, Vaghi MA: Intracranial neurinomas of the nerves of the jugular foramen. Report of 12 personal cases. Acta Neurochir 31:201-221, 1975

31. Pollock BE, Kondziolka D, Flickinger JC, et al: Preservation of cranial nerve function after radiosurgery for nonacoustic schwannomas. Neurosurgery 33:597-601, 1993

32. Samii M, Migliori M, Tatagiba M, et al: Surgical treatment of trigeminal schwannomas. J Neurosurg 82:711-718, 1995 


\section{Treatment of dumbbell-shaped jugular foramen schwannomas}

33. Sasaki T, Takakura K: Twelve cases of jugular foramen neurinoma. Skull Base Surg 1:152-160, 1991

34. Suzuki F, Handa J, Todo G: Intracranial glossopharyngeal neurinomas. Report of two cases with special emphasis on computed tomography and magnetic resonance imaging findings. Surg Neurol 31:390-394, 1989

35. Tan LC, Bordi L, Symon L, et al: Jugular foramen neuromas: a review of 14 cases. Surg Neurol 34:205-211, 1990

36. Vellutini EA, Cruz OL, Velasco OP, et al: Reversible hearing loss from cerebellopontine angle tumors. Neurosurgery 28: 310-313, 1991
37. Yamakami I, Nakamura T, Ono J, et al: Recovery of hearing after removal of a large jugular foramen schwannoma: report of two cases. Surg Neurol 51:60-65, 1999

Manuscript received June 15, 2004.

Accepted in final form July 5, 2004.

Address reprint requests to: Ossama Al-Mefty, M.D., 4301 West Markham, \#507, Little Rock, Arkansas 72205. email: aekeeland@ uams.edu. 